\title{
ESTUDO COMPARATIVO DOS EFEITOS DAS CORRENTES AUSSIE, INTERFERENCIAL E ESTIMULAÇÃO ELÉTRICA NERVOSA TRANSCUTÂNEA (TENS) NO TRATAMENTO DA LOMBALGIA CRÔNICA
}

\author{
COMPARATIVE STUDY OF THE EFFECTS OF AUSSIE CURRENT, INTERFERENTIAL AND \\ TRANSCUTANEOUS NERVOUS ELECTRIC STIMULATION (TENS) IN THE TREATMENT OF \\ CHRONIC LOW BACK PAIN
}

DOI: $10.16891 / 2317-434 X . v 10 . e 1 . a 2022 . p p 1260-1263$

Recebido em: 10.11.2021 | Aceito em: 10.11.2021

\section{Irma Bantim Felício Calou, Bruna Estéffany Pereira Mota Duarte e Albério Ambrósio Calvacante}

Centro Universitário Doutor Leão Sampaio

\section{RESUMO}

Dentre as principais algias da coluna, destaca-se a lombalgia crônica inespecífica, condição de grande potencial incapacitante. Dentre as diversas estratégias de intervenção, a eletroterapia promove analgesia e melhora da função. As principais correntes analgésicas são: a Estimulação Elétrica Nervosa Transcutânea (TENS), a Corrente Interferencial (C.I.) e a Corrente Aussie. Teve-se como objetivo comparar os efeitos dessas três correntes no tratamento da lombalgia crônica inespecífica. Trata-se de um estudo experimental (ensaio clinico randomizado), analítico e de abordagem quantitativa. A pesquisa envolveu 32 participantes, divididos em 4 grupos; o grupo 1 (G1): submetido à aplicação da corrente TENS, o grupo 2 (G2): C. I., o grupo 3 (G3): Corrente Aussie e o grupo 4 (G4): controle/placebo. A dor foi avaliada antes e depois da terapia, através da escala visual analógica (EVA) da dor, associada ao questionário de incapacidade Roland-Morris. Resultados mostraram significância estatística para redução da dor, para todas as correntes: TENS $\left(p=0,000^{*}\right)$, Corrente Interferencial $\left(p=0,000^{*}\right)$ e Aussie $\left(p=0,000^{*}\right)$; o grupo placebo não obteve resultado significativo $(p=0,03)$. A comparação dos valores de dor, após o uso da escala EVA, ao final da conduta, demonstra que a média de intensidade da dor é de 0,$38 ; 1,13 ; 1$ e 3,75 para as correntes interferencial, Aussie, TENS e para o placebo, respectivamente. Com isso, afirmamos que a eletroterapia analgésica demonstra resultados positivos na redução da dor lombar crônica, não havendo diferença significativa entre as correntes. Sugere-se mais estudos, com maior número de sessões e N amostral para obtenção de maior nível de significância.

Palavras-chave: Dor Lombar; Modalidade de Fisioterapia; Terapia por estimulação elétrica.

\section{ABSTRACT}

Among the main pains of the spine, chronic nonspecific low back pain stands out, a condition with great disabling potential. Among the various intervention strategies, electrotherapy promotes analgesia and improves function. The main analgesic currents are: Transcutaneous Electrical Nerve Stimulation (TENS), Interferential Current (C.I.) and Aussie Current. The objective was to compare the effects of these three currents in the treatment of nonspecific chronic low back pain. This is an experimental study (randomized clinical trial), analytical and with a quantitative approach. The research involved 32 participants, divided into 4 groups; group 1 (G1): submitted to the application of TENS current, group 2 (G2): C.I., group 3 (G3): Aussie current and group 4 (G4): control/placebo. Pain was assessed before and after therapy, using a visual analogue pain scale (VAS) associated with the Roland-Morris disability questionnaire. Results showed statistical significance for pain reduction for all currents: TENS ( $p=0.000 *$ ), Interferential Current $\left(p=0.000^{*}\right)$ and Aussie $\left(p=0.000^{*}\right)$; the placebo group did not obtain a significant result $(p=0.03)$. Comparison of pain values after using the VAS scale at the end of the procedure shows that the mean pain intensity is $0.38 ; 1.13 ; 1$ and 3.75 for interferential, Aussie, TENS and placebo currents, respectively. Thus, we affirm that analgesic electrotherapy demonstrates positive results in reducing chronic low back pain, with no significant difference between the currents. Further studies are suggested, with a greater number of sessions and $\mathrm{N}$ sample to obtain a higher level of significance.

Keyword: Low Back Pain; Type of Physiotherapy; Electrical stimulation therapy. 
V.10 N.1 (2022) ISSN: 2317-434X

\section{INTRODUÇÃO}

Atualmente, as disfunções da coluna lideram as causas de dor, sofrimento e de incapacidade em vários países do mundo. Na Europa, 27\% dos trabalhadores queixam-se de dor na coluna e $23 \%$ queixam-se de dores musculares (EUROPEAN AGENCY FOR SAFETY AND HEALTH AT WORK, 2010).

O número de brasileiros que são incapacitados pela lombalgia crônica (LC) é muito alto. Os relatórios estimam que aproximadamente 10 milhões de pessoas no Brasil são afetadas (SALVETTI et al., 2012). A LC representa a principal razão para solicitações de benefícios por incapacidade e é a terceira causa mais comum de aposentadoria relacionada às deficiências no Brasil (MEZIAT; SILVA, 2011). É uma condição que pode atingir até $65 \%$ das pessoas anualmente e até $84 \%$ das pessoas em algum momento da vida (WALKER, 2000).

O tratamento de LC compreende uma gama de diferentes estratégias de intervenção, incluindo cirurgia, terapia medicamentosa e reabilitação multidisciplinar (MIDDELKOOP et al., 2011). Os fisioterapeutas usam uma ampla gama de intervenções terapêuticas conservadoras e não-farmacológicas, algumas das quais são amplamente recomendadas em várias diretrizes (FALAVIGNA et al., 2016). Os principais objetivos são reduzir a dor e melhorar a capacidade funcional (DEROSA; PORTERFIELD, 1992; TULDER; KOES; BOMBARDIER, 2002).

Em relação às modalidades de fisioterapia, a Eletroterapia é um método que objetiva, entre outros aspectos, a analgesia e melhora da função através de um aumento da energia (elétrica, sonora, luminosa ou térmica) no corpo (PAGE et al., 2016). Dentre essas modalidades, tem-se a estimulação elétrica nervosa transcutânea (TENS) que é um recurso não invasivo, de fácil aplicação, que pode ser utilizado em pacientes jovens, adultos e idosos, com o objetivo de induzir analgesia (PENA; BARBOSA; ISHIKAWA, 2008). A corrente interferencial, que tem efeito na diminuição da intensidade da dor, aumentando significativamente o limiar da dor e pode até mesmo bloquear a condução nervosa (DEFRIN; ARIEL; PERETZ; 2005; WARD; TOUMBOUROU; MCCARTHY, 2009). A corrente Aussie (também chamada de corrente Australiana) por sua vez, é uma nova geração de corrente elétrica para estimulação motora e sensorial (analgésica), com vantagens sobre os tradicionais métodos de estimulação.

\section{METODOLOGIA}

Esta pesquisa trata-se de um estudo intervencionista, baseado em um ensaio clinico randomizado, analítico e de abordagem quantitativa. Foi realizada em uma Instituição de Ensino Superior de Juazeiro do Norte - Ceará.

A amostra foi do tipo intencional, onde a condição comum a todos os participantes do estudo é o fato de possuírem lombalgia crônica. Foi composta por 32 pessoas, divididos igualitariamente em quatro grupos. A definição de quem participou de cada grupo foi efetuada mediante sorteio, onde foram distribuídos 32 papeis de cores equivalentes aos seus grupos. $\mathrm{O}$ paciente sem saber a que se refere o sorteio, puxou em uma caixa um papel, onde foi revelado apenas ao terapeuta a corrente a ser aplicada, o que garante à randomização do processo.

Essa pesquisa foi submetida e cadastrada a plataforma Brasil, e após, submetida e aprovada pelo comitê de ética, sobe o número do parecer consubstanciado do CEP 2.376.862.

Foram inclusos no estudo, pessoas entre 18 a 65 anos, com dor na região lombar há pelo menos 3 meses, o que assegura a cronicidade da sintomatologia dolorosa, e com qualificação da dor em nível $\geq 3$ segundo a Escala Visual Analógica (EVA) da dor. Foram excluídos do estudo, aqueles que apresentaram presença de infecções em geral, ferimentos na região lombar, doenças dermatológicas, hipertensão descompensada, alergia ao gel eletrocondutor, placas metálicas na região lombar, cardiopatas, usuários de marca-passo cardíaco, mulheres gestantes ou com suspeita, portadores de neoplasias, e pessoas em estado febril.

Cada participante recebeu uma única aplicação, e esta conduta foi realizada no momento em que o participante referiu dor.

Para avaliação do paciente, foi utilizada uma ficha de avaliação de autoria dos pesquisadores, onde constam os dados pessoais do participante, anamnese e perguntas pertinentes ao estado clínico. Foi aplicado o questionário de Incapacidade Roland-Morris (Roland-Morris Disability Questionnaire - RMDQ) adaptado pelos pesquisadores, que avalia a interferência da lombalgia nas atividades laborais da vida diária. A pontuação é obtida através da soma dos itens, que variam de zero (sem incapacidade) a 24 (incapacidade severa), valores superiores a 14 pontos indicam incapacidade física, porém como o questionário foi adaptado,

os valores foram calculados proporcionalmente aos do original, onde zero indica (sem incapacidade), 10 (incapacidade severa), valores superiores a 6 indicam incapacidade física. Também foi utilizada a Escala Visual 


\section{V.10 N.1 (2022) ISSN: 2317-434X}

Analógica (EVA), para quantificar a dor varia de 0 a 10, sendo 0 a ausência de dor e 10 a pior dor. Dessa forma, de 1-3 é considerada dor leve; de 4-6, moderada; de 7-9 dor forte. A melhora de dois pontos é considerada um bom resultado.

Tabela 1. Protocolos de aplicação de corrente utilizados no estudo

\begin{tabular}{|c|c|c|c|c|}
\hline GRUPO & $\begin{array}{l}\text { CORRENTE } \\
\text { APLICADA } \\
\end{array}$ & MODO DE APLICAÇÃO & PARÂMETROS & TEMPO \\
\hline G1 & TENS (IBRAMED) & $\begin{array}{l}\text { Foram utilizados os eletrodos em } \\
\text { silicone, acoplados na região } \\
\text { lombar, especificamente onde o } \\
\text { participante indicou a localização } \\
\text { da dor. }\end{array}$ & $\begin{array}{l}\text { Modo BURST com } \\
\text { frequências de trens de pulso } \\
\text { de } 2 \text { a } 5 \mathrm{~Hz} \text {, frequência } \\
\text { portadora de } 250 \mathrm{~Hz} \text { e largura } \\
\text { de pulso de } 300 \mathrm{us} \text {. }\end{array}$ & 30 minutos \\
\hline G2 & $\begin{array}{l}\text { INTERFERENCIAL } \\
\text { (IBRAMED) }\end{array}$ & $\begin{array}{l}\text { Foram utilizados os eletrodos em } \\
\text { silicone, acoplados na região } \\
\text { lombar, especificamente onde o } \\
\text { participante indicou a localização } \\
\text { da dor. }\end{array}$ & $\begin{array}{c}\text { Modo tetrapolar, de varredura, } \\
\text { utilizando-se do programa } \\
\text { slope quadrado } 1: 1 \text {, com } \\
\text { frequência portadora de } \\
2000 \mathrm{~Hz} \text {, amplitude } \\
\text { moduladora de frequência } \\
\text { AMF base de } 100 \mathrm{~Hz} \text { e } \Delta \mathrm{AMF} \\
\text { de } 50 \mathrm{~Hz} \text {. }\end{array}$ & 30 minutos \\
\hline G3 & AUSSIE (IBRAMED) & $\begin{array}{c}\text { Foram aplicados os eletrodos em } \\
\text { silicone, de forma cruzada, na } \\
\text { região lombar onde o paciente } \\
\text { referia dor. }\end{array}$ & $\begin{array}{c}\text { Frequência portadora de } \\
\text { 4KHz, frequência de } \\
\text { modulação de } 70 \mathrm{~Hz} \text { com } \\
\text { duração de largura de pulso de } \\
\text { 4ms. }\end{array}$ & 30 minutos \\
\hline G4 & GRUPO CONTROLE & $\begin{array}{l}\text { Foram submetidas à colocação } \\
\text { dos eletrodos com posicionamento } \\
\text { correto para aplicação. Porém, o } \\
\text { aparelho foi ligado apenas por } 30 \\
\text { segundos e teve a }\end{array}$ & $\begin{array}{l}\text { Corrente TENS no modo } \\
\text { BURST com frequências de } \\
\text { trens de pulso de } 2 \text { a } 5 \mathrm{~Hz}, \\
\text { com uma frequência portadora } \\
\text { de } 250 \mathrm{~Hz} \text { e uma largura de } \\
\text { pulso de } 300 \text { us (por } 30 \\
\text { segundo). }\end{array}$ & 30 minutos \\
\hline
\end{tabular}

Fonte: Próprios Autores, 2021.

\section{RESULTADOS}

A amostra foi composta por 32 pessoas, que foram divididas igualmente em 4 grupos, grupo TENS (G1), grupo interferencial (G2), grupo Aussie (G3) e grupo controle (G4), com média de idade de 27,22 $\pm(8,89)$, sendo dessas, 8 pessoas do sexo masculino $(25 \%)$ e 24 do sexo feminino (75\%). A maioria apresentando dor na região da lombar baixa $(59,40 \%)$. A dor é sentida em

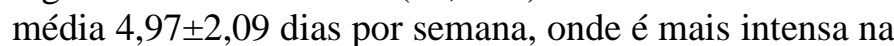
posição sentada com $46,90 \%$ e em repouso com $71,90 \%$. A quantificação da dor segundo a escala da EVA antes e após a aplicação das correntes, foi relatada com média inicial de 5,91 $\pm 1,33$ e média final de $1,56 \pm 1,85$, com valor significativo no Te ste $\mathrm{t}$ de Student para amostras pareadas de $\mathrm{P}=0,000^{*}$.

A EVA antes e depois da aplicação de cada corrente, onde foi expresso o valor de significância utilizando o mesmo método estatístico da anterior. Afirma-se que as correntes utilizadas foram igualmente significativas, todos com com $\mathrm{p}=0,000$. $\mathrm{O}$ grupo placebo não foi significante com $\mathrm{p}=0,03$. Sobre a comparação da EVA final entre os grupos de intervenção e o placebo, a média de intensidade da dor é de 0,38 para a interferencial, 1,13 para a corrente Aussie, de 1 para a TENS e de 3,75 para o placebo, representando que todos foram mais eficientes na redução da dor em relação ao placebo

Já na relação entre o questionário de Rolland Morris e a EVA, mostrando que, existe uma relação linear entre os dois parâmetros, porém, trata-se de uma correlação fraca, conforme o método de Pearson, em que, para $\mathrm{R}$ maior que 0 e menor que 0,3 , temos uma correlação fraca, ou seja, o resultado do questionário varia pouco com relação ao resultado da EVA.

Comparando o resultado final da diminuição da dor segundo a escala da EVA, foi utilizado o teste Anova 


\section{V.10 N.1 (2022) ISSN: 2317-434X}

One-Way que compara a distribuição de três ou mais grupos em amostras independentes. $\mathrm{O}$ teste indicou que não há diferenças estatisticamente significativas entre as médias das correntes, sendo as três igualmente efetivas na diminuição da dor, porém o grupo placebo não se mostrou significante em relação a essa variável.

\section{CONCLUSÃO}

Através da apresentação dos dados do presente estudo conclui-se que os recursos eletroterapêuticos como a TENS, Corrente Interferencial e Corrente Aussie causaram grande alívio de dor lombar crônica nos participantes, considerando que as médias de intensidade da dor antes da aplicação da TENS foi de 6,13 reduzindo para 1,00 após intervenção, C.I foi de 5,75 diminuindo para 0,38 , corrente Aussie foi de 6,38 antes da intervenção para 1,13 após intervenção, grupo placebo foi de 5,38 antes para 3,775 depois, obtivendo resultados estatisticamente significantes com $\mathrm{G} 1\left(\mathrm{p}=0,000^{*}\right), \mathrm{G} 2$ $\left(\mathrm{p}=0,000^{*}\right), \mathrm{G} 3\left(\mathrm{p}=0,000^{*}\right)$, onde o placebo $(\mathrm{G} 4)$ não obteve significância com $(\mathrm{p}=0,03)$.

Comparando os três recursos não obteve-se diferenças estatisticamente significantes, concluindo então que os três recursos são bons para a redução da dor lombar, não encontrando um que seja considerado melhor que o outro, porém as três correntes sobressaem o placebo.

Em virtude de serem encontradas poucas pesquisas na área, sugere-se mais estudos sobre o tema tratado, principalmente em relação à corrente Aussie, com maior número de sessões e $\mathrm{N}$ amostral, para obtenção de maior nível de significância, buscando enfatizar melhor as suas eficácias.

\section{REFERÊNCIAS}

DEFRIN, R.; ARIEL, E.; PERETZ, C. Estimulação elétrica nociva versus inócua segmentar para o alívio da dor crônica e o efeito da sensação de desvanecimento durante o tratamento. Dor. v. 115, n.1-2, p. 152-160, 2005.

DEROSA, C. P.; PORTERFIELD, J. A. Um modelo de fisioterapia para o tratamento da dor lombar. PhysTher. v. 72, n.4, p. 269-272, 1992.

DOHNERT, M. B.; BAUER, J. P.; PAVÃO, T. S. Study of the effectiveness of interferential current as compared to transcutaneous electrical nerve stimulation in reducing chronic low back pain. Dor. v.16 n.1, 2015.

EUROPEAN AGENCY FOR SAFETY AND HEALTH AT WORK. European surve of enterprises on new and emerging riscksmonaging safety and health at work. luxembourgpuplications office of the european union, 2010.

FALAVIGNA, A. A. L.; TELES, A. R.; BRAGA, G. L.; BARAZZETTI, D. O.; FEITOSA, A. S. A.;LOPES, J. B.;ELOISA BONFA, E.;ARI SR HALPERN, A. S. R.A prospective study predicting the outcome of chronic low back pain and physical therapy: the role of fear-avoidance beliefs and extraspinal pain. Rev. Bras. Reumatol. São Paulo, v.56, n.5, p. 384-390, 2016.

MEZIAT F, N.; SILVA, G. A. Pensão de invalidez por dores nas costas entre os beneficiários da segurança social. Rev Saude Publica, Brasil,v. 45, p. 494-502, 2011.
MIDDELKOOP, M. V.; RUBINSTEIN, S. M.; KUIJPERS, T.; VERHAGEN, A. P.;OSTELO, R.; KOES, B. W.; TULDER, M. W. V. A systematic review on the effectiveness of physical and rehabilitation interventions for chronic non-specific low back pain. European Spine Journal. v. 20, n.1, p. 19-39, 2011.

PAGE, M. J.; GREEN, S.; MROCKI, M. A.; SURACE, S. J.; DEITCH, J.; MCBAIN, B.; LYTTLE, N.; BUCHBINDER, R. Electrotherapy modalities for rotator cuff disease. Cochrane Data base of Systematic Reviews, 2016.

PENA, R.; BARBOSA, L. A.; ISHIKAWA, N. M. Estimulação elétrica transcutânea do nervo (TENS) na dor oncológica - uma revisão da literatura. Rev Bras Cancerol. v. 54, n.2, p. 193-199, 2008.

RAIMUNDO, A.K.S; ARAÚJO, V.M. Estudo comparativo do efeito analgésico das frequências de base da corrente interferencial na lombalgia por osteoartrose. Fisioterapia Brasil. v.9, n.4, p.269-274, 2008.

SALVETTI, M. D. E. G.; PIMENTA, C. A.; BRAGA, P. E.; CORRÊA, C. F. Disability related to chronic low back pain: prevalence and associated factors. RevEscEnferm USP. v. 46, p. 16-23, 2012.

WALKER, B. F. The prevalence of low back pain: a systematic review of the literature from 1966 to 1998. J SpinalDisord. v. 13 , p. $205-217,200$ o. 